\title{
Primary and secondary basis set superposition error at the SCF and MP2 levels: $\mathrm{H}_{3} \mathrm{~N}--\mathrm{Li}^{+}$and $\mathrm{H}_{2} \mathrm{O}--\mathrm{Li}^{+}$
}

\author{
Zdzis/aw Latajka ${ }^{a)}$ and Steve Scheiner ${ }^{b), c)}$ \\ Department of Chemistry and Biochemistry, Southern Illinois University, Carbondale, Illinois 62901
}

(Received 9 March 1987; accepted 1 April 1987)

\begin{abstract}
The primary basis set superposition error (BSSE) results from the artificial lowering of the energy of each subunit of a pair by the presence of "ghost orbitals" of its partner. In addition, these ghost orbitals perturb the one-electron properties of the molecule, causing a change in the interaction energy, an effect known as secondary BSSE which is not corrected by the counterpoise procedure. The primary and secondary BSSE are calculated for the interactions of $\mathrm{NH}_{3}$ and $\mathrm{H}_{2} \mathrm{O}$ with $\mathrm{Li}^{+}$, using a variety of different basis sets. It is found that the $2^{\circ} \mathrm{BSSE}$ can be quite large, comparable in magnitude to the $1^{\circ}$ component at both the SCF and MP2 levels. There is no basis found for the supposition that $2^{\circ}$ BSSE improves the calculated interaction energy, nor do the $1^{\circ}$ and $2^{\circ}$ effects cancel one another in general. While the MP2 BSSE tends to be smaller than the SCF analog, the former can be similar in magnitude to the "true" MP2 contribution to the interaction; failure to remove the BSSE can hence lead to a qualitatively incorrect interpretation of the effects of electron correlation. Comparison with a system in which basis set superposition is rigorously excluded suggests that subtraction of both the full $1^{\circ}$ and $2^{\circ}$ BSSE is appropriate and does not overcorrect the potential. Addition of a diffuse $s p$ shell, especially if coupled with orbital exponent reoptimization, leads to a lowering of the $1^{\circ}$ and $2^{\circ} \mathrm{BSSE}$, which moreover take on opposite sign and cancel one another to some extent.
\end{abstract}

\section{INTRODUCTION}

A full understanding of the macroscopic properties of the gaseous, liquid, and solid states requires an understanding of the forces contributing to the potential energy surface of pairs of interacting molecules. Ab initio theoretical methods $^{1}$ can in principle provide a wealth of information inaccessible by current experimental techniques. Because the basis sets describing each subunit are finite, calculations of intermolecular interactions are subject to a problem termed basis set superposition error (BSSE). ${ }^{2}$ Specifically, as the two molecules approach one another, the basis set of one subunit is "enlarged" by the presence of the orbitals of its partner, lowering its molecular energy via the variation principle. This stabilization is purely a mathematical artifact which would not be present if the basis set of each subunit were complete in the first place. The magnitude of the superposition error can be quite appreciable when compared to the true interaction energy, distorting the latter quantity and obscuring interpretation of the calculated data.

A means of evaluating and correcting the primary BSSE, known as the Boys and Bernardi functional counterpoise procedure, ${ }^{2}$ involves calculating the energy of each monomer within the framework of the basis set of the entire complex. The difference between this quantity, which includes the effects of the partner's "ghost orbitals," and the energy of the isolated monomer represents the artificial stabilization due to these extra orbitals. Although there has

\footnotetext{
a) Permanent address: Institute of Chemistry, University of Wroclaw, 50383 Wroclaw, Poland.

b) Address correspondence to this author.

${ }^{c}$ Recipient of Research Career Development Award from NIH.
}

been some past controversy in the literature as to whether all the ghost orbitals should be included or some subset thereof, it is now generally accepted that the full set is necessary, at least at the SCF level..$^{3-17}$ The question of whether the counterpoise correction provides a good measure of the BSSE at correlated levels, on the other hand, has only recently begun to attract attention and a full consensus has not yet been reached. ${ }^{5,18-31}$ Nonetheless, this matter is a crucial one as the correlated BSSE has been found to be quite large, comparable in magnitude to the SCF BSSE and to the true correlation contribution to the interaction energy. Moreover, whereas the BSSE can be lowered to negligible proportions by modest basis set improvements at the SCF level, the correlated BSSE appears extremely resilient to such tactics. ${ }^{5,24-29}$

In addition to lowering the energy of the molecule, the ghost orbitals of its partner subunit will in general also produce perturbations in the molecule's electronic properties, e.g., its dipole moment. ${ }^{10,26,32-35}$ These altered properties will in turn produce a change in the interaction energy, an effect which is not addressed by the standard counterpoise correction and which may be termed "secondary" BSSE. ${ }^{32}$ Based on their calculations of the water dimer with a minimal basis set, Karlström and Sadlej had originally suggested the secondary BSSE may improve the calculated interaction energy since the ghost orbitals can enhance the accuracy of the molecular properties. ${ }^{32}$ However, other calculations using fairly large basis sets seem to indicate that on the contrary, the results are improved when secondary BSSE is removed. ${ }^{10,33-35}$ Unfortunately, the latter work dealt exclusively with very weak van der Waals complexes and direct comparison with the stronger hydrogen bond may not be appropriate. It is this question of secondary BSSE to which we address our attention here.

Because of the myriad of properties that may be affected 
by ghost orbitals, the actual evaluation of the energetic consequences of secondary BSSE can be quite complex. For this reason, it is necessary to choose a simple and tractable model system for study - one in which the secondary BSSE can be evaluated in a straightforward manner. The interaction between $\mathrm{NH}_{3}$ and $\mathrm{Li}^{+}$is ideal in this regard. Due to the compact nature of the $\mathrm{Li}^{+}$cation, requiring only a fairly small basis set for an adequate description, one can expect the ghost orbitals of the $\mathrm{NH}_{3}$ to have little or no effect on $\mathrm{Li}^{+}$(an assumption borne out by the calculations described below). As a result, only one molecule of the pair is affected by ghost orbitals, drastically simplifying the analysis. In addition, use of a spherical cation like $\mathrm{Li}^{+}$eliminates several terms of the electrostatic expansion, leaving only ion-dipole, ion-quadrupole, etc. The $\mathrm{Li}^{+}$cation's very low polarizability further simplifies matters by minimizing terms such as dipole $\left(\mathrm{NH}_{3}\right)$-induced dipole $\left(\mathrm{Li}^{+}\right)$. Finally, since $\mathrm{Li}^{+}$ contains only two electrons, both of which are in its $1 s$ orbital, use of the MP2 frozen-core approximation precludes any excitation of the $\mathrm{Li}^{+}$electrons. This greatly facilitates our analysis of the effects of correlation since there is no intermolecular correlation term and the only intramolecular component remaining involves the $\mathrm{NH}_{3}$ molecule alone. By carrying out analogous calculations on the $\mathrm{H}_{2} \mathrm{O}--\mathrm{Li}^{+}$system, it is possible to draw conclusions concerning the generality of our results.

This work has several objectives. We intend to estimate the magnitude of secondary BSSE and make a comparison with the primary effect. Another important comparison concerns the relative magnitudes of the SCF and correlated BSSEs. Questions we address include whether secondary BSSE is in fact an improvement and under what conditions might $2^{\circ}$ and $1^{\circ}$ superposition errors cancel one another. By examining a number of basis sets, varied in a systematic manner, we clearly identify the characteristics of these sets which are responsible for the BSSE and provide a set of criteria to be used in choosing a basis set for study of molecular interactions.

Section II details our procedures for evaluating the $1^{\circ}$ and $2^{\circ}$ BSSE and describes the various basis sets. The molecular properties to which these basis sets lead are compared in the next section. The basis set superposition errors are reported in Sec. IV, followed by a detailed analysis of the interaction at long range. Section VI reports a comparison with $\mathrm{H}_{2} \mathrm{O}-\mathrm{Li}^{+}$. The results are summarized and discussed in the last section.

\section{COMPUTATIONAL PROCEDURES}

$A b$ initio calculations were carried out using the GAUSSIAN-80 codes. $^{36}$ Second-order M 6 ller-Plesset perturbation theory (MP2), ${ }^{37}$ keeping frozen the cores of first-row atoms, was used to evaluate the effects of electron correlation. SCF and MP2 contributions to the dipole moment and dipole polarizability tensor of $\mathrm{NH}_{3}$ were computed by the finite-field perturbation method ${ }^{38}$ : numerical differentiation based on a parabolic fit of the energy with respect to an applied electric field, ${ }^{39}$ the magnitude of which $( \pm 0.005$ a.u.) was chosen so as to produce inaccuracies in the dipole moment and polarizability of less than $10^{-4}$ and $10^{-2}$ a.u., respectively.

Interaction energies were computed as the difference in total energy between each complex and the reference subunits at infinite separation. The counterpoise procedure ${ }^{2}$ was used to evaluate the primary basis set superposition error ( $1^{\circ}$-BSSE). The counterpoise correction is equal to the difference in energy between the isolated subunits on one hand and the subunit energies, each calculated within the basis set and geometry of the entire complex, on the other. The secondary basis set superposition error in any property of either monomer is defined as the change in this property caused by introduction of the ghost orbitals of the partner subunit:

$$
\Delta P_{\mathrm{A}}=P_{\mathrm{A}}(\mathrm{AB})-P_{\mathrm{A}}(\mathrm{A}) \text {, }
$$

where $P_{\mathrm{A}}$ refers to the property of interest of subunit $\mathrm{A}$ and the extent of the basis set is indicated by the subunits listed in the following parentheses.

The geometry of the $\mathrm{NH}_{3}$ molecule was held fixed in its experimentally determined structure ${ }^{40}: r(\mathrm{NH})=1.0124 \AA$ and $\theta(\mathrm{HNH})=106.68^{\circ}$. The $\mathrm{Li}^{+}$cation approached $\mathrm{NH}_{3}$ along the $C_{3}$ symmetry axis of the latter molecule.

Most of the basis sets examined here are modifications of the standard 6-31G** which includes a single set of $p$ functions on $\mathrm{H}$ and $d$ functions on first-row atoms. ${ }^{41}$ In summary, "dif" designates replacement of the rather large 6-31G** polarization function exponents $\left[\alpha_{d}(N)=0.80\right.$; $\left.\alpha_{p}(H)=1.1\right]$ by more diffuse functions with exponents 0.25 and 0.15 , respectively. The latter orbitals were recommended by van Duijneveldt-van de Rijdt and van Duijneveldt $^{42}$ as providing a better description of one-electron properties. As an alternative to changing these orbital exponents, $2 d$ indicates instead the addition of a second set of $d$ functions $\left(\alpha=0.25\right.$ ) to $\mathrm{N}$. $\mathrm{Li}^{+}$was described by the standard $6-31 \mathrm{G}^{* *}$ for all three of the above sets. Addition of a diffuse $s p$ shell to $\mathrm{N}(\alpha=0.10)$ is denoted by + . Other modifications, described in detail in an earlier work, ${ }^{27}$ were designed to reduce the spurious effects of basis set superposition by optimization of all exponents within the context of each monomer subunit rather than the individual atoms as in the original basis set. $+V P^{S}$ and $+V P^{M}$ refer to the $6-31+G^{* *}$ set (including diffuse sp shell on $N$ ), reoptimized at the SCF and MP2 levels, respectively. Addition of a second set of $d$ functions to $\mathrm{N}$, also with exponent optimized, is denoted $+V P^{s}(2 d)^{s}$ or $+V P^{M}(2 d)^{M}$, with the superscripts having the same meaning as above. In all the above sets which include a diffuse $s p$ shell on $N$, a similar shell was added to $\mathrm{Li}^{+}$as well $(\alpha=0.007)$. For purposes of comparison, we also include the standard triple-valence $6-311 \mathrm{G}^{* *}$ basis set in our calculations. ${ }^{43}$

\section{MONOMER PROPERTIES}

Prior to reporting the interaction energies and superposition errors in the $\mathrm{H}_{3} \mathrm{~N}--\mathrm{Li}^{+}$complex, we list in Table I the relevant properties of the isolated $\mathrm{NH}_{3}$ subunit calculated with each of our basis sets. As may be observed by comparison of the experimental dipole moment of $1.47 \mathrm{D}$ with the data in the first column, all basis sets overestimate the dipole moment of $\mathrm{NH}_{3}$ at the SCF level. The smallest degree of 
TABLE I. Calculated dipole moment and dipole polarizability components" of $\mathrm{NH}_{3}$.

\begin{tabular}{|c|c|c|c|c|c|c|c|c|c|}
\hline & \multicolumn{3}{|c|}{$\mu, \mathbf{D}$} & \multicolumn{3}{|c|}{$\alpha_{z z}$, a.u. } & \multicolumn{3}{|c|}{$\alpha_{x x}$, a.u. } \\
\hline & SCF & MP2 & SCF + MP2 & SCF & MP2 & $\mathrm{SCF}+\mathrm{MP} 2$ & SCF & MP2 & $\mathrm{SCF}+\mathrm{MP2}$ \\
\hline $6-31 G^{* *}$ & 1.891 & -0.045 & 1.846 & 5.68 & 0.09 & 5.77 & 9.44 & -0.03 & 9.41 \\
\hline dif & 1.574 & -0.120 & 1.453 & 10.10 & 1.14 & 11.24 & 11.98 & 0.69 & 12.67 \\
\hline $2 d$ & 1.503 & -0.083 & 1.420 & 10.32 & 0.97 & 11.29 & 12.01 & 0.60 & 12.61 \\
\hline $6-311 G^{* *}$ & 1.768 & -0.053 & 1.715 & 7.48 & 0.37 & 7.85 & 9.88 & 0.07 & 9.95 \\
\hline+ & 1.840 & -0.050 & 1.789 & 9.85 & 1.37 & 11.22 & 9.91 & 0.12 & 10.02 \\
\hline$+V P^{S}$ & 1.842 & -0.051 & 1.791 & 10.08 & 1.43 & 11.51 & 9.80 & 0.08 & 9.88 \\
\hline$+V P^{M}$ & 1.813 & -0.048 & 1.764 & 9.90 & 1.17 & 11.06 & 9.79 & 0.08 & 9.87 \\
\hline$+V P^{S}(2 d)^{S}$ & 1.705 & -0.039 & 1.666 & 10.80 & 1.31 & 12.11 & 10.58 & 0.15 & 10.72 \\
\hline$+V P^{M}(2 d)^{M}$ & 1.740 & -0.029 & 1.710 & 10.16 & 1.06 & 11.22 & 10.14 & 0.10 & 10.23 \\
\hline Ref. $44^{b}$ & 1.619 & -0.095 & 1.524 & 13.32 & 2.42 & 15.74 & 12.76 & 0.98 & 13.74 \\
\hline Expt. & & & $1.471^{c}$ & & & 14.5 & & & \\
\hline
\end{tabular}

$z$ axis is coincident with $C_{3}$ rotation axis of $\mathrm{NH}_{3}$.

b $(12 s 8 p 3 d 1 f / 7 s 2 p 1 d)$ contracted to [8s5p3d $1 f / 4 s 2 p 1 d]$.

'From Ref. 45.

dFrom Ref. 46; value corresponds to rotational average $\left(\alpha_{x x}+\alpha_{y y}+\alpha_{z z}\right) / 3$.

exaggeration is associated with the dif and $2 d$ basis sets in the second and third rows, wherein the $\mathrm{N}$ center contains a particularly diffuse set of $d$ orbitals. It should be noted from the penultimate row that even the very large basis set of Diercksen and Sadlej ${ }^{44}$ overestimates the dipole moment by $10 \%$ at the SCF level.

Second-order perturbation theory reduces the dipole moment with all basis sets, as is evident from the next column of Table I. With a sufficiently large basis set, the MP2 corrections are capable of reproducing the experimental value to within $4 \%$. The largest MP2 reductions are associated with the dif and $2 d$ basis sets, leading to very slight underesti-

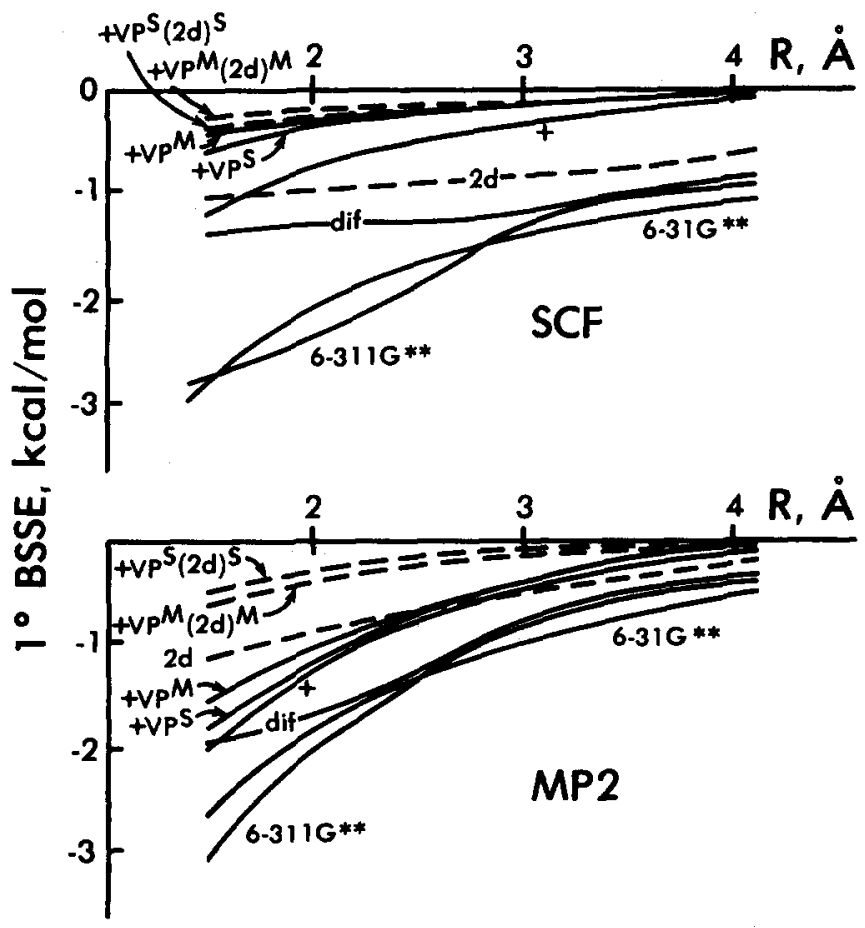

FIG. 1. Primary BSSE at the (a) SCF and (b) MP2 levels calculated by the counterpoise procedure for $\mathrm{H}_{3} \mathrm{~N}--\mathrm{Li}^{+}$as a function of the distance between $\mathrm{Li}$ and $\mathrm{N}$ centers. Broken curves are used to distinguish results for basis sets containing two sets of $d$ functions. mates of the experimental value, errors of only $1 \%$ and $3 \%$, respectively. The moments calculated with the remainder of the basis sets remain too large, even after MP2 correction.

Turning now to the polarizability tensor, it is clear that the SCF estimates of the components both parallel $\left(\alpha_{z z}\right)$ and perpendicular $\left(\alpha_{x x}\right)$ to the $C_{3}$ axis of $\mathrm{NH}_{3}$ are too low. The standard 6-31 $\mathrm{G}^{* *}$ and $6-311 \mathrm{G}^{* *}$ sets are particularly bad in this regard, with larger polarizabilities arising from introduction of diffuse functions of either $d$ or sp type on N. With only one minor exception, MP2 contributions raise the polarizability, especially the $z$ component. Such correlationinduced increases are consistent with prior calculations ${ }^{47}$; as a result, the rotationally averaged MP2 polarizability calculated by Diercksen and Sadlej with their large basis set is within $1 \%$ of the experimental value. All the smaller sets underestimate the polarizability by a substantial margin.

The best available calculations indicate the parallel component of the polarizability exceeds the perpendicular element with a ratio $\alpha_{z z} / \alpha_{x x}=1.15$ at the MP2 level. ${ }^{44} \mathrm{~A}$ ratio larger than unity is characteristic of all our basis sets in which a diffuse $s p$ shell has been added to $\mathbf{N}$. In contrast, those basis sets lacking these functions predict a parallel component smaller than the perpendicular element.

\section{BASIS SET SUPERPOSITION ERROR}

\section{A. Primary BSSE}

As $\mathrm{Li}^{+}$approaches $\mathrm{NH}_{3}$, its orbitals extend the incomplete basis set of $\mathrm{NH}_{3}$, leading to the well known basis set superposition error characteristic of molecular interactions. By employing the functional counterpoise procedure of Boys and Bernardi, ${ }^{2}$ one can extract the primary BSSE, which is illustrated as a function of $R(\mathrm{~N}-\mathrm{Li})$ for each of our basis sets in Fig. $1 .^{48}$ In all cases, the primary BSSE is negative, exaggerating the attractive force, and approaches zero asymptotically for large intermolecular separation. Note that the BSSE contained within the second-order correlation, illustrated in Fig. 1(b), is comparable in magnitude to the SCF BSSE in Fig. 1 (a). At either level, the standard 6-31G** and 6-311G** sets lead to the largest primary BSSE, particularly 

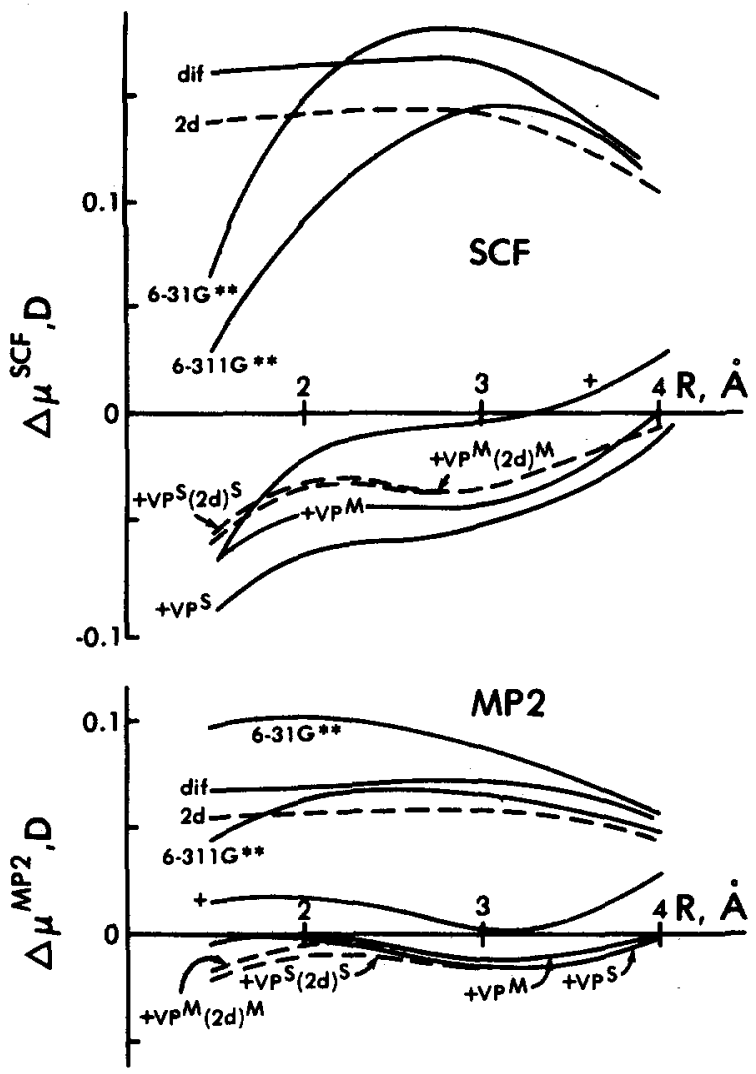

FIG. 2. Change in dipole moment of $\mathrm{NH}_{3}$ caused by presence of $\mathrm{Li}^{+}$ghost orbitals centered a distance $R$ from $\mathbf{N}$ atom.

for small $\boldsymbol{R}$. At the SCF level, the diffuse $d$ functions contained within the dif and $2 d$ basis sets diminish the BSSE to some extent but the greatest reduction is associated with addition of the + functions, especially when the orbital exponents are optimized. A similar pattern is noted for the MP2 BSSE except that failure to include two sets of $d$ functions on the optimized basis sets increases their BSSE somewhat.

\section{B. Molecular properties}

Concomitant with the stabilization of $\mathrm{NH}_{3}$ arising from the incorporation of the $\mathrm{Li}^{+}$ghost orbitals into its own basis set is a perturbation of the $\mathrm{NH}_{3}$ electronic distribution. One manifestation of this aspect of basis set superposition is a change in the dipole moment of $\mathrm{NH}_{3}$ caused by the presence of the $\mathrm{Li}^{+}$ghost orbitals. This change, denoted $\Delta \mu$ relative to the moment of the isolated $\mathrm{NH}_{3}$ molecule, is illustrated in Fig. 2 as a function of $R(N-L i)$. Unlike the primary BSSE which is always negative due to the variation principle, since the additional orbitals of the partner subunit must result in an energy decrease, there is no such law which applies to the dipole moment; hence, $\Delta \mu$ may be of either sign. In confirmation, $\Delta \mu$ is positive for some basis sets and negative for others, even switching its sign at a particular value of $\boldsymbol{R}$ for some sets.

Focusing our attention first on the SCF results depicted in Fig. 2(a), the basis sets fall into one of two groups. The standard $6-31 \mathrm{G}^{* *}$ and $6-311 \mathrm{G}^{* *}$, as well as the $2 d$ and dif variants, all lead to positive values of $\Delta \mu$. That is, the presence of the ghost orbitals of $\mathrm{Li}^{+}$acts to increase the dipole
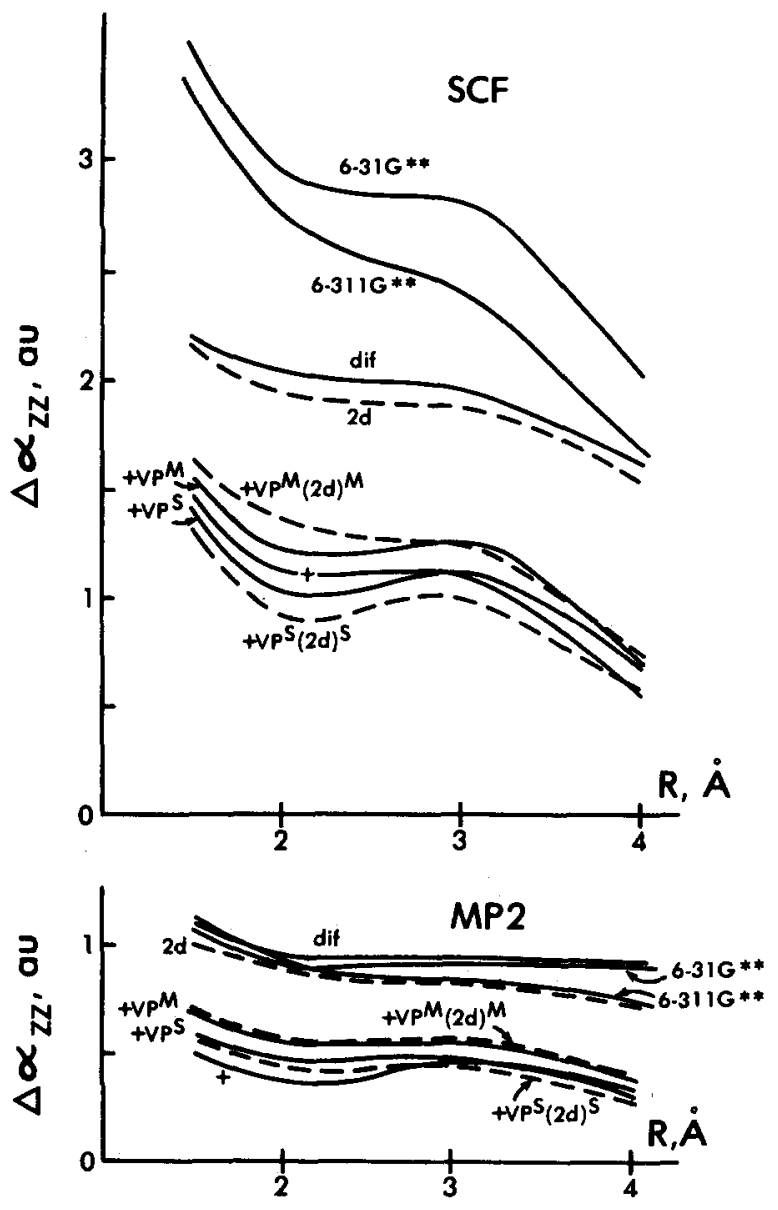

FIG. 3. Increase in component of dipole polarizability tensor along $C_{3}$ axis of $\mathrm{NH}_{3}$ caused by $\mathrm{Li}$ ghost orbitals.

moment of $\mathrm{NH}_{3}$. In the first two cases, this quantity peaks in the vicinity of $3 \AA$, while the others plateau in the range $1.5<R<3 \AA$. On the other hand, $\Delta \mu$ is generally negative for the other basis sets, all containing a diffuse $s p$ shell, i.e., the moment of $\mathrm{NH}_{3}$ is lessened by basis set superposition when the latter shell is included in the basis set. $\Delta \boldsymbol{\mu}$ appears to become more positive as $R$ increases for these sets.

The patterns observed for the MP2 changes in the dipole moment, reported in Fig. 2(b), are fairly similar although the magnitude of $\Delta \mu$ is somewhat smaller at this level. On the other hand, the absolute contribution of second-order correlation to the dipole moment is also much smaller than the SCF component (see Table I); hence, the superposition effects contained in Fig. 2(b) represent a major factor at the MP2 level, proportionately even more so than errors in the SCF segment. Indeed, the magnitude of $\Delta \mu^{M P 2}$ for the standard $6-31 \mathrm{G}^{* *}$ and $6-311 \mathrm{G}^{* *}$ sets is even larger than the "true" MP2 contribution to $\mu$ in Table I and of opposite sign. Failure to correct for this secondary BSSE could therefore lead to a qualitatively incorrect conclusion concerning the effects of electron correlation upon the interaction. (This point is explored in greater detail below.) The much smaller values of $\Delta \mu^{\text {MP2 }}$ observed for the sets containing the + functions would tend to enhance the appropriateness of these sets for such investigations.

Like the dipole moment, the polarizability of $\mathrm{NH}_{3}$ is also subject to basis set superposition effects. The enhance- 


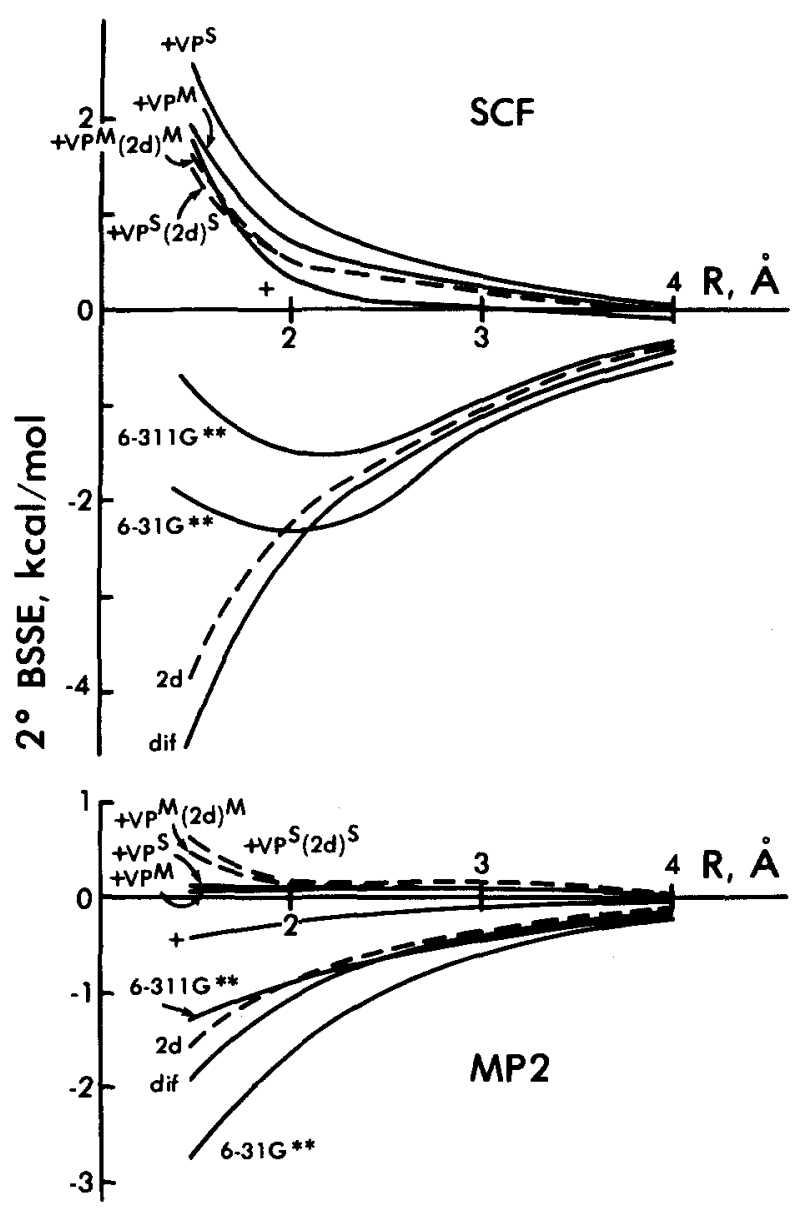

FIG. 4. Secondary BSSE for $\mathrm{H}_{3} \mathrm{~N}-\mathrm{Li}^{+}$due to change in $\mathrm{NH}_{3}$ dipole moment caused by $\mathrm{Li}$ ghost orbitals; computed by Eq. (1).

ment of the parallel component of the polarizability due to the ghost orbitals of $\mathrm{Li}^{+}, \Delta \alpha_{\mathrm{zz}}$, is presented in Fig. 3(a) for the SCF level and Fig. 3(b) for MP2. As already noted for the dipole moment, the change in the polarizability resulting from basis set superposition is clearly larger at the SCF level than MP2; nonetheless, the magnitudes of these effects are far from negligible in either case. For example, the SCF value of $\alpha_{z z}$ in Table I for the 6-31G** basis set is increased $48 \%$ when the $\mathrm{Li}^{+}$ghost orbitals are situated $3 \AA$ from $\mathrm{NH}_{3}$; the analogous MP2 increase is many times larger than the "true" MP2 component of 0.09 a.u. The basis sets fall into the same two categories with regard to the polarizability as enunciated earlier for the dipole moment: those sets containing a diffuse $s p$ shell lead to substantially smaller superposition errors in the polarizability as compared to the standard sets, even when diffuse $d$ functions are included in the latter.

Although not included in Fig. 3, the superposition error in the perpendicular component $\alpha_{x x}$ was calculated as well. As might be expected on the basis of the positioning of the ghost orbitals along the $z$ axis, the BSSE in $\alpha_{x x}$ is several times smaller than $\Delta \alpha_{z z}$. Just as for the parallel component, the SCF BSSE in $\alpha_{x x}$ is considerably larger than the MP2 equivalent. The increases in the dipole polarizability tensor noted here are consistent with prior work using considerably larger basis sets.

\section{Secondary BSSE}

In order to examine how these superposition errors in the molecular properties of $\mathrm{NH}_{3}$ can influence the calculated interaction energy with $\mathrm{Li}^{+}$, we note first that the principal interaction between these two species is electrostatic, with the leading term of ion-dipole type. Hence, the most important contribution to the secondary basis set superposition error may be taken as the Coulombic interaction between a unit positive charge at the $\mathrm{Li}$ position and the change in the dipole moment of $\mathrm{NH}_{3}$ induced by the presence of the $\mathrm{Li}$ ghost orbitals, $\Delta \mu$ :

$$
2^{\circ} \mathrm{BSSE}=-e \Delta \mu / d^{2},
$$

where $d$ is the distance between the Li nucleus and the $\mathrm{NH}_{3}$ center of mass.

Equation (1) represents only the first term in the multipole expansion of the secondary BSSE. Other components would consider the effects of ghost orbitals upon the quadrupole and higher moments of $\mathrm{NH}_{3}$. However, these terms would die off more quickly with increasing intermolecular separation than the ion-dipole expression. Although ghost orbitals were shown above to have a substantial influence upon the polarizability of $\mathrm{NH}_{3}$, this property contributes little to the secondary BSSE because of the $1 / R^{4}$ dependence of the charge-polarizability term. We therefore expect Eq. (1) to embody the major contribution to the interaction energy of the changes in the $\mathrm{NH}_{3}$ electronic properties induced by the Li ghost orbitals, particularly at large values of $\boldsymbol{R}$.

These secondary BSSEs are presented as a function of distance $R$ in Fig. 4. As in the prior figures, 4(a) contains the SCF results while MP2 data are illustrated in 4(b). The SCF dipole moment increases indicated by the positive signs of $\Delta \mu$ in Fig. 2(a) for the standard $6-31 G^{* *}$ and $6-311 G^{* *}$ basis sets, as well as the dif and $2 d$ variants, lead to a strengthened interaction with $\mathrm{Li}^{+}$and hence to the negative values for the secondary BSSE in Fig. 4(a) for these basis sets. Due to the inverse quadratic dependence in Eq. (1), the magnitudes of the secondary SCF BSSE tend to large values as $R$ diminishes. (The exceptions are the $6-31 \mathrm{G}^{* *}$ and 6-311G** sets where $\Delta \mu^{\text {SCF }}$ drops precipitously for $R<3$ A.) However, one would not apply Eq. (1) as an accurate measure of secondary BSSE at this close range anyway due to the divergence of the multipole expansion.

The remaining sets, all of which contain a diffuse $s p$ shell, yield positive secondary SCF BSSEs due to the decreases in the SCF dipole moment associated with the $\mathrm{Li}^{+}$ ghost orbitals. Just as the MP2 values of $\Delta \mu$ are generally much smaller than their SCF counterparts, similar relationships apply to the secondary BSSEs in Fig. 4. One feature worthy of particular note is the much reduced BSSEs at both the SCF and MP2 levels arising from addition of the + orbitals to the basis sets, particularly if coupled to exponent optimization.

Comparison of Figs. 1 and 4 reveals that the secondary BSSE can be comparable to, and in many cases larger than, the primary BSSE. Whereas the latter term is always attractive, the secondary BSSE can take either sign. It is therefore hazardous in the general case to expect these two types of 
TABLE II. Contributions to the interaction energy of $\mathrm{Li}^{+}$with $\mathrm{H}_{n} \mathrm{X}$ for $R=4.0 \AA$ including corrections for $1^{\circ}$ and $2^{\circ}$ BSSE, compared to interaction of $\mathrm{H}_{n} \mathrm{X}$ with a point charge (PC). All entries in $\mathrm{kcal} / \mathrm{mol}$.

\begin{tabular}{|c|c|c|c|c|c|c|c|c|}
\hline & \multicolumn{4}{|c|}{ SCF } & \multicolumn{4}{|c|}{ MP2 } \\
\hline & \multicolumn{3}{|c|}{$\mathbf{H}_{3} \mathrm{~N}--\mathrm{Li}^{+}$} & \multirow[b]{2}{*}{$\mathbf{H}_{3} \mathbf{N}-\mathbf{P C}$} & \multicolumn{3}{|c|}{$\mathrm{H}_{3} \mathrm{~N}--\mathrm{Li}^{+}$} & \multirow[b]{2}{*}{$\mathbf{H}_{3} \mathbf{N}-\mathbf{P C}$} \\
\hline & none & $1^{\circ}$ & $1^{\circ}+2^{\circ}$ & & none & $1^{\circ}$ & $1^{\circ}+2^{\circ}$ & \\
\hline $\begin{array}{l}\text { 6-31G** } \\
\text { dif } \\
2 d \\
6-311 G^{* *} \\
+ \\
+V P^{S} \\
+V P^{M} \\
+V P^{S}(2 d)^{S} \\
+V P^{M}(2 d)^{M}\end{array}$ & $\begin{array}{l}-13.01 \\
-11.64 \\
-11.23 \\
-12.40 \\
-11.88 \\
-11.63 \\
-11.52 \\
-11.15 \\
-11.26\end{array}$ & $\begin{array}{r}-11.97 \\
-10.86 \\
-10.60 \\
-11.48 \\
-11.81 \\
-11.60 \\
-11.49 \\
-11.12 \\
-11.17\end{array}$ & $\begin{array}{l}-11.36 \\
-10.38 \\
-10.17 \\
-11.03 \\
-11.71 \\
-11.65 \\
-11.50 \\
-11.16 \\
-11.18\end{array}$ & $\begin{array}{r}-10.75 \\
-9.76 \\
-9.56 \\
-10.43 \\
-11.35 \\
-11.46 \\
-11.25 \\
-10.92 \\
-10.95\end{array}$ & $\begin{array}{l}-0.79 \\
-0.51 \\
-0.43 \\
-0.65 \\
-0.34 \\
-0.24 \\
-0.24 \\
-0.17 \\
-0.17\end{array}$ & $\begin{array}{r}-0.26 \\
-0.12 \\
-0.16 \\
-0.19 \\
-0.19 \\
-0.14 \\
-0.15 \\
-0.10 \\
-0.15 \\
N\end{array}$ & $\begin{array}{r}-0.02 \\
0.12 \\
0.02 \\
-0.00 \\
-0.16 \\
-0.15 \\
-0.15 \\
-0.12 \\
-0.15 \\
\end{array}$ & $\begin{array}{r}0.19 \\
0.38 \\
0.26 \\
0.20 \\
-0.10 \\
-0.06 \\
-0.02 \\
-0.09 \\
-0.09\end{array}$ \\
\hline \multirow{2}{*}{$+V P^{M}(2 d)^{M}$} & \multicolumn{3}{|c|}{$\mathrm{H}_{2} \mathrm{O}--\mathrm{Li}^{+}$} & & \multicolumn{3}{|c|}{$\mathrm{H}_{2} \mathrm{O}--\mathrm{Li}^{+}$} & \\
\hline & none & $1^{\circ}$ & $1^{\circ}+2^{\circ}$ & $\mathrm{H}_{2} \mathrm{O}-\mathrm{PC}$ & none & $1^{\circ}$ & $1^{\circ}+2^{\circ}$ & $\mathrm{H}_{2} \mathrm{O}-\mathrm{PC}$ \\
\hline $\begin{array}{l}\text { 6-31G** } \\
\text { dif } \\
+ \\
\text { Ext }\end{array}$ & $\begin{array}{r}-10.53 \\
-9.60 \\
-10.04 \\
-8.93\end{array}$ & $\begin{array}{r}-9.82 \\
-9.02 \\
-10.01 \\
-8.88\end{array}$ & $\begin{array}{r}-9.55 \\
-8.82 \\
-10.00 \\
-8.87\end{array}$ & $\begin{array}{l}-9.43 \\
-8.70 \\
-9.96 \\
-8.81\end{array}$ & $\begin{array}{r}-0.18 \\
0.28 \\
0.06 \\
0.33\end{array}$ & $\begin{array}{l}0.19 \\
0.55 \\
0.14 \\
0.38\end{array}$ & $\begin{array}{c}0.31 \\
0.65 \\
0.09 \\
\cdots\end{array}$ & $\begin{array}{l}0.33 \\
0.69 \\
0.15 \\
0.42\end{array}$ \\
\hline
\end{tabular}

[ $[6 s 4 p 3 d / 4 s 2 p]$ contracted from $(8 s 4 p 3 d / 5 s 2 p)$ basis set of Ref. 50.

superposition error to cancel one another if left uncorrected. We note that the presence of + functions, especially if coupled to exponent reoptimization, leads to much lower BSSE than the remaining basis sets at all levels: SCF and MP2, primary as well as secondary. In these cases, it also happens that the opposite signs of the primary and secondary BSSEs lead to large-scale cancellation.

It was indicated earlier that the ghost orbitals can have a dramatic impact upon the calculated contribution of electron correlation to the dipole moment. In order to provide some estimate of the energetic consequences, let us consider the distance $R=2 \AA$ as an example. With the standard 6-31G** basis set, the MP2 contribution to the interaction energy, before introduction of any corrections, is -1.40 $\mathrm{kcal} / \mathrm{mol}$. By comparison, the secondary BSSE computed by Eq. (1) is $-1.62 \mathrm{kcal} / \mathrm{mol}$. It is hence clear that failure to subtract the latter error will yield a falsely attractive term when in reality the correlation component is slightly repulsive. (Subtraction of the primary BSSE of $-1.77 \mathrm{kcal} / \mathrm{mol}$ will further add to the repulsive character.) Turning now to the $+V P^{S}$ set, differing from $6-31 \mathrm{G}^{* *}$ only by addition of + functions on $\mathbf{N}$ and reoptimization of exponents, the uncorrected MP2 component is +0.65 , already repulsive. The secondary BSSE is only $0.04 \mathrm{kcal} / \mathrm{mol}$ and hence could probably be neglected (although the primary BSSE of - 1.18 remains important). We conclude that secondary BSSE represents a serious potential problem which can be alleviated to a great extent by careful selection of basis set.

\section{LONG-RANGE INTERACTIONS}

At long range, the dominant contribution to the interaction between a base like $\mathrm{NH}_{3}$ and $\mathrm{a} \mathrm{Li}{ }^{+}$cation is electrostat- ic. This term, as well as smaller contributions from induction, exchange repulsion, etc., are contained within the SCF portion of the interaction energy. The first column of data in Table II lists the SCF interaction energies computed for a distance of $4 \AA$ for each of our basis sets. After subtraction of the primary BSSE, the interactions become somewhat less attractive, as is evident from the next column. Subtraction of the secondary BSSE as well leads to our final corrected SCF interaction energies in the following column.

At long distance, one might expect a positively charged point charge to mimic very closely the appearance of a $\mathrm{Li}^{+}$ cation. The lack of electrons around the point charge removes the possibility of any exchange repulsion but this term should be vanishingly small at a distance of $4 \AA$. The lack of electrons also negates any charge deformability but this should also introduce only a small error since the polarizability of $\mathrm{Li}^{+}$is quite small $\left(0.027 \AA^{3}\right) .^{49}$ What is most important about the interaction with a point charge is that the absence of orbitals about the latter entity precludes the possibility of any basis set superposition error and may thus be used as a convenient benchmark by which to gauge methods for correction of this error.

Particular insights may thus be gleaned by comparing the $\mathrm{H}_{3} \mathrm{~N}$-point charge interactions in Table II with the preceding column where both primary and secondary superposition errors have been subtracted from the $\mathrm{H}_{3} \mathrm{~N}-\mathrm{Li}^{+}$interaction. In no case do corrections for the latter errors lead to an interaction that is less attractive than the point charge value. This finding contradicts previous statements that the counterpoise procedure overcorrects the interaction and supports the contention that this procedure offers a reasonable means of correction. The slightly more attractive nature of the fully corrected $\mathrm{H}_{3} \mathrm{~N}-\mathrm{Li}^{+}$interaction is probably due 
TABLE III. Calculated dipole moment and dipole polarizability along $C_{2}$ axis of $\mathrm{H}_{2} \mathrm{O}$.

\begin{tabular}{|c|c|c|c|c|c|c|}
\hline & \multicolumn{3}{|c|}{$\mu, \bar{D}$} & \multicolumn{3}{|c|}{$\alpha_{z z}$, a.u. } \\
\hline & SCF & MP2 & $\mathrm{SCF}+\mathrm{MP} 2$ & SCF & MP2 & $\mathrm{SCF}+\mathrm{MP2}$ \\
\hline $6-31 G^{* *}$ & 2.184 & -0.088 & 2.096 & 5.11 & -0.23 & 4.88 \\
\hline dif & 1.967 & -0.188 & 1.779 & 7.28 & 0.85 & 8.13 \\
\hline+ & 2.284 & -0.046 & 2.239 & 5.86 & 0.37 & 6.23 \\
\hline Ext & 1.950 & -0.131 & 1.819 & 8.28 & 1.02 & 9.30 \\
\hline Ref. $52^{b}$ & 1.983 & -0.132 & 1.851 & 8.51 & 1.26 & 9.77 \\
\hline Expt. & & & $1.840^{c}$ & & & $9.62^{d}$ \\
\hline
\end{tabular}

a $[6 s 4 p 3 d / 4 s 2 p]$ contracted from $(8 s 4 p 3 d / 5 s 2 p)$ basis set of Ref. 50 .

${ }^{b}(12 s 8 p 3 d 1 f / 7 s 2 p 1 d)$ contracted to [8s5p3d $\left.1 f / 4 s 2 p 1 d\right]$.

c From Ref. 53.

dFrom Ref. 54.

to terms such as dipole-induced dipole which do not appear in the point charge expression. Another factor may be the higher polarizability of $\mathrm{NH}_{3}$ in the presence of the $\mathrm{Li}$ ghost orbitals, or to their effects on the quadrupole and higher moments of $\mathrm{NH}_{3}$, secondary BSSE which is not removed by Eq. (1).

The MP2 contribution to the interaction energy can in general be partitioned into intra- and intermolecular terms. In this particular system, the frozen core approximation which leaves the two $\mathrm{Li}^{+}$electrons uncorrelated eliminates intermolecular terms, e.g., dispersion. Intramolecular correlation affecting the $\mathrm{Li}$ cation is likewise precluded. The only remaining term is of intramolecular type, arising from the interaction between uncorrelated $\mathrm{Li}^{+}$and the correlationinduced changes in the electronic properties of $\mathrm{NH}_{3}$. Hence, replacement of the $\mathrm{Li}^{+}$species with a point charge, containing neither electrons nor orbitals, should ideally reproduce the effects of correlation upon the $\mathrm{H}_{3} \mathrm{~N}--\mathrm{Li}^{+}$system, particularly at long intermolecular separations. The absence of orbitals on the point charge again precludes the possibility of basis set superposition, furnishing a numerical test of any procedure for removing primary and secondary BSSE.

The calculated MP2 contribution to the interaction energy between $\mathrm{NH}_{3}$ and $\mathrm{Li}^{+}$at $R=4 \AA$ is reported in the first column on the right side of Table II where it may be noted that an attractive correlation effect is associated with all basis sets, although the magnitude of this term is quite variable. The next column lists the same data after correction for the primary BSSE by the counterpoise procedure, which leads in all cases to a somewhat less attractive component. Inclusion of the $1^{\circ}$ correction also improves the level of agreement from one basis set to the next, substantially reducing the range of values.

Following subsequent subtraction of the secondary BSSE, evaluated via Eq. (1), the long-range MP2 interactions become even less attractive and in certain cases slightly repulsive, as indicated by the penultimate column. Even after correction for both primary and secondary BSSE, the computed MP2 interactions for $\mathrm{H}_{3} \mathrm{~N}--\mathrm{Li}^{+}$remain slightly more attractive in all cases than the $\mathrm{H}_{3} \mathrm{~N}$-point charge system listed in the last column. It is therefore apparent that at the MP2 level, just as for SCF, removal of both the full counterpoise correction (including all ghost orbitals, occupied as well as vacant) and the secondary BSSE does not lead to an overcorrected, overly repulsive interaction.

The remaining discrepancy between the last two columns does not appear to be due to polarizability changes induced in $\mathrm{NH}_{3}$ by $\mathrm{Li}^{+}$ghost orbitals since the energetic consequence of the secondary BSSE due to polarizability was computed at $R=4 \AA$ and found to be numerically insignificant. Instead, the difference is probably due principally to a secondary BSSE associated with the quadrupole moment of $\mathrm{NH}_{3}$, and possibly to higher-order moments as well. What should be emphasized is that inclusion of secondary (as well as primary) BSSE appears to substantially improve the results, enhancing the agreement with the $\mathrm{H}_{3} \mathrm{~N}$-point charge data where basis set superposition is clearly not a factor. This conclusion, based on the very strong $\mathrm{H}_{3} \mathrm{~N}--\mathrm{Li}^{+}$ interaction, is entirely consistent with earlier findings in which removal of both primary and secondary BSSE improved the very weak interactions in van der Waals complexes. ${ }^{10,33-35}$

\section{VI. $\mathrm{H}_{2} \mathrm{O}-\mathrm{-Li}^{+}$}

Calculations analogous to those reported above have also been carried out for the interaction of $\mathrm{Li}^{+}$with $\mathrm{H}_{2} \mathrm{O}$. In the latter system, we have restricted ourselves to a comparison of the standard $6-31 \mathrm{G}^{* *}$ set, its dif variant where the polarization function exponents have been decreased to $\alpha_{d}(\mathrm{O})=0.25$ and $\alpha_{p}(\mathrm{H})=0.15$, and the + set where a diffuse $s p$ shell with orbital exponent 0.10 has been added to the standard 6-31G** set of oxygen. As a benchmark, we have also made use of a much more extended basis set, denoted Ext, which includes three sets of $d$ functions on $O$ and two sets of $p$ functions on $\mathrm{H}^{50}$ The water molecule was held frozen in its experimental geometry ${ }^{5 !}: r(\mathrm{OH})=0.957 \AA$; $\theta(\mathrm{HOH})=104.5^{\circ}$; and $\mathrm{Li}^{+}$placed along its $C_{2}$ symmetry axis.

The molecular properties of $\mathrm{H}_{2} \mathrm{O}$ reported in Table III obey patterns quite similar to those noted above for $\mathrm{NH}_{3}$ (see Table I). As before, all basis sets exaggerate the dipole moment at the SCF level, particularly $6-31 \mathrm{G}^{* *}$ and + . Again, second-order perturbation theory lowers the dipole in all cases, with the largest decrement associated with the dif basis set. The resulting moment with the latter basis is rather close to experiment, albeit slightly too small, while the remaining sets considerably overestimate the moment. Following inclusion of second-order correlation, the larger basis 


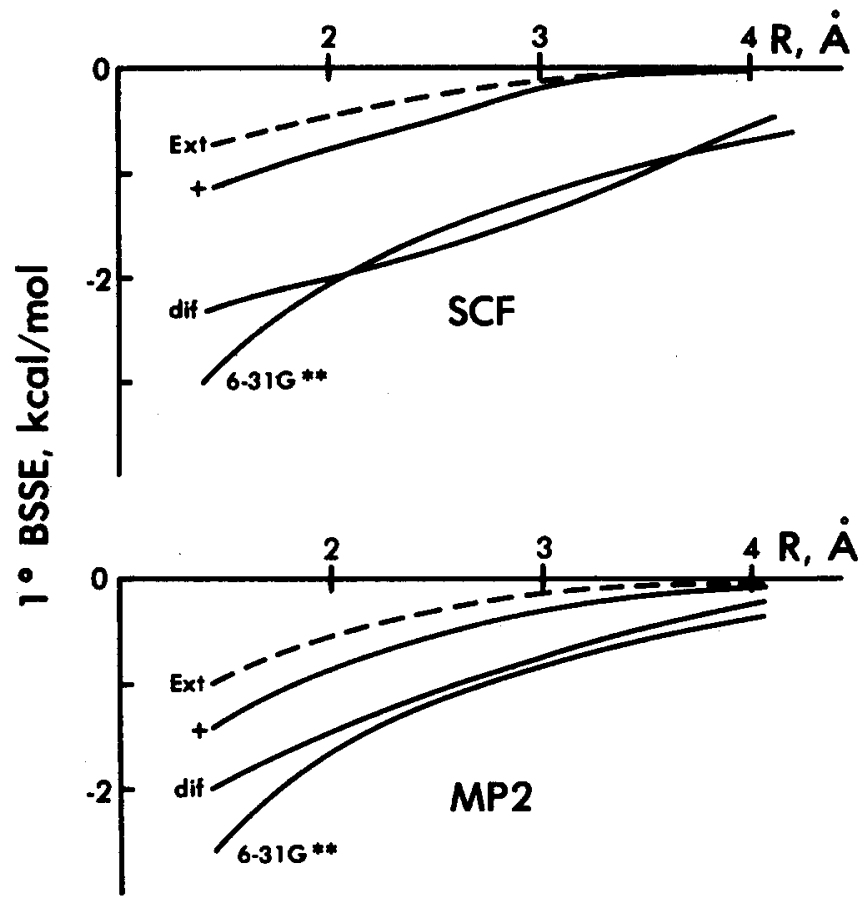

FIG. 5. Primary BSSE calculated for $\mathrm{H}_{2} \mathrm{O}--\mathrm{Li}^{+}$.

sets come rather close to experiment, particularly the very large basis set of Diercksen et al., ${ }^{32}$ which is within $0.6 \%$ of experiment.

All of the SCF polarizabilities are too small compared to
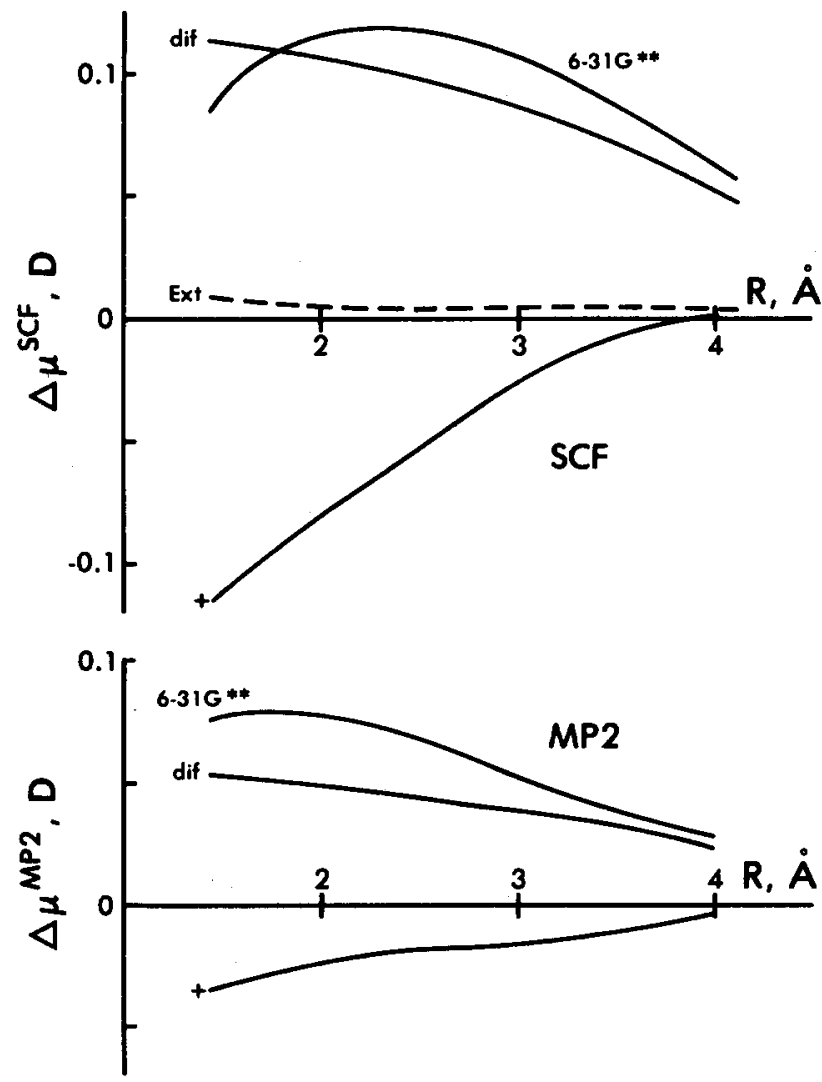

FIG. 6. Change in $\mathrm{H}_{2} \mathrm{O}$ dipole moment caused by presence of $\mathrm{Li}^{+}$ghost orbitals centered a distance $R$ from $O$ center.
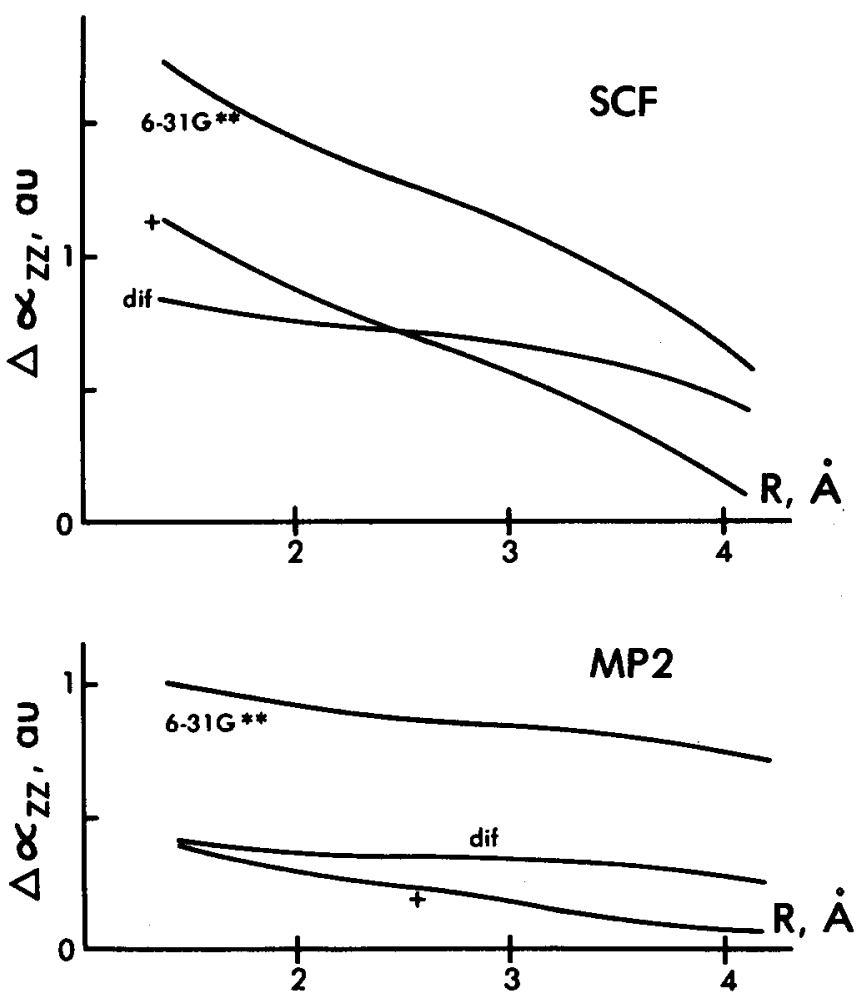

FIG. 7. Increase in component of dipole polarizability tensor along $C_{2}$ axis of $\mathrm{H}_{2} \mathrm{O}$ caused by $\mathrm{Li}$ ghost orbitals.

experiment, again consistent with $\mathrm{NH}_{3}$. Even after adding the MP2 contribution which is positive (with the exception of 6-31G**), the polarizability remains somewhat too small, with the dif basis being least in error. The data in the last several rows of Table III suggest that the polarizability can be adequately described at the SCF + MP2 level, provided a sufficiently flexible basis set is employed.

The primary BSSE illustrated in Fig. 5 is consistent with the $\mathrm{H}_{3} \mathrm{~N}--\mathrm{Li}^{+}$data in that the MP2 counterpoise correction is comparable in magnitude to the SCF values. Reducing the polarization function exponents has little effect on the 6-31G** BSSE, whereas addition of the diffuse sp shell markedly lowers this quantity, especially at the SCF level. Extension of the basis set to a fairly large size further lowers the $1^{\circ} \mathrm{BSSE}$, although it is certainly not negligible at either level, as indicated by the dashed curves in Fig. 5 .

The effects of the $\mathrm{Li}$ ghost orbitals upon the calculated dipole moment of $\mathrm{H}_{2} \mathrm{O}$ are displayed in Fig. 6 where patterns reminiscent of Fig. 2 are evident. Within the context of the 6$31 \mathrm{G}^{* *}$ set and its dif variant, the dipole moment is enhanced by these orbitals while the opposite effect is observed when a set of + functions is included in the oxygen basis set. In contrast, the ghost orbitals of $\mathrm{Li}$ have little effect upon the dipole moment of $\mathrm{H}_{2} \mathrm{O}$ with the Ext basis set. Once again, the superposition error in the MP2 contribution to the dipole moment is comparable in certain cases to the true MP2 component.

Comparison of Figs. 3 and 7 indicates that the polarizability of $\mathrm{H}_{2} \mathrm{O}$ is somewhat less susceptible to secondary BSSE than is that of $\mathrm{NH}_{3}$. As in the case of $\mathrm{NH}_{3}$, the ghost orbitals produce a much larger increase in the $6-31 \mathrm{G}^{* *}$ po- 


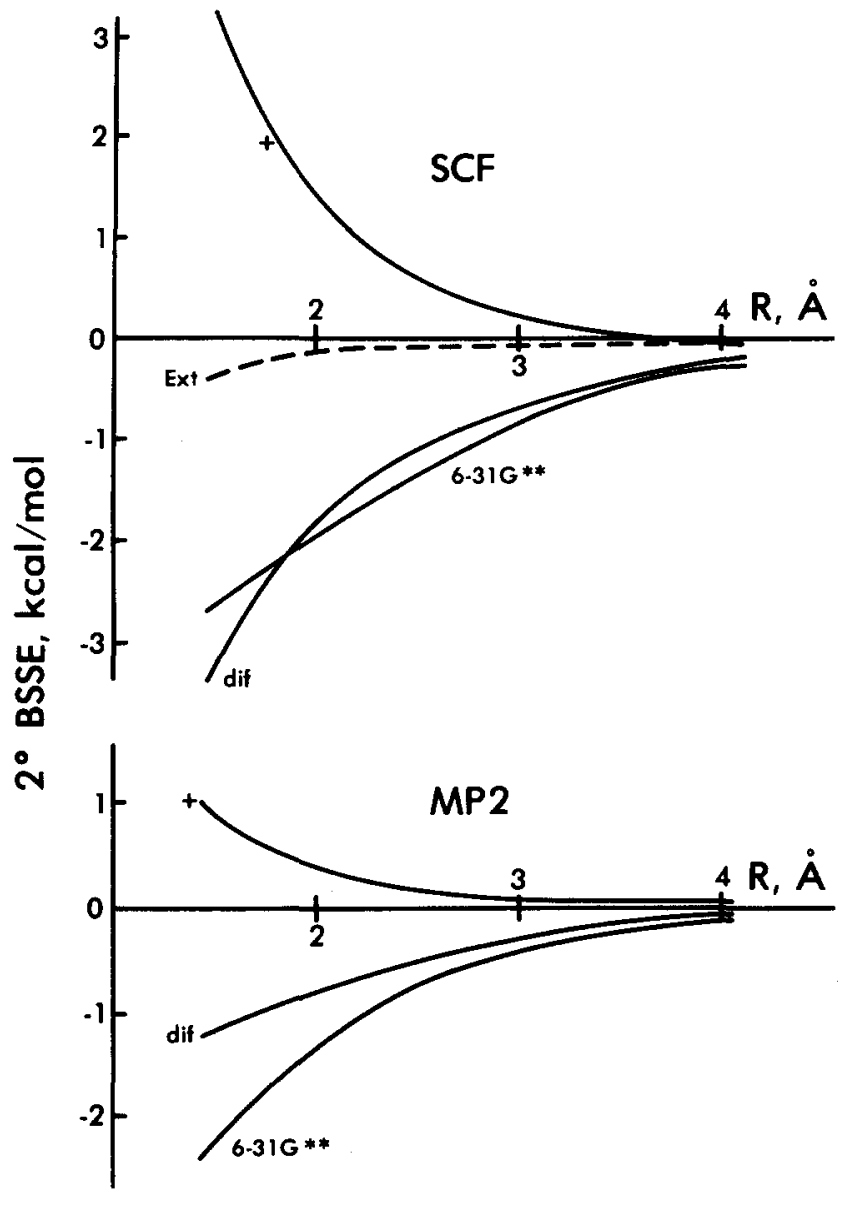

FIG. 8. Secondary BSSE for $\mathrm{H}_{2} \mathrm{O}--\mathrm{Li}^{+}$computed by Eq. (1).

larizability of $\mathrm{H}_{2} \mathrm{O}$, as compared to the + set. The decrease of the polarization function exponents (dif set) appears to more effectively reduce the effects of ghost orbitals upon the polarizability of $\mathrm{H}_{2} \mathrm{O}$ than in the case of $\mathrm{NH}_{3}$.

Turning finally to the secondary BSSE, again calculated by Eq. (1), the patterns for $\mathrm{H}_{2} \mathrm{O}--\mathrm{Li}^{+}$in Fig. 8 are similar to the trends for the $\mathrm{NH}_{3}$ analog in Fig. 4 in that the 6-31G** and dif results are quite close to one another, and negative at both the SCF and MP2 levels. The SCF secondary $\mathrm{BSSE}$ for the + basis is positive for $\mathrm{H}_{2} \mathrm{O}-\mathrm{Li}^{+}$although somewhat larger in magnitude than for $\mathrm{H}_{3} \mathrm{~N}--\mathrm{Li}^{+}$. The sign of the MP2 $2^{\circ}$ BSSE is positive for the former system and negative for the latter; most important though, the magnitude of this quantity is much smaller in either case than for the 6-31G** or dif sets. The SCF $2^{\circ}$ BSSE calculated for the Ext basis set is extremely small, consistent with the insensitivity of the corresponding dipole moment to ghost orbitals.

The SCF and MP2 components of the interaction energy of $\mathrm{H}_{2} \mathrm{O}$ with $\mathrm{Li}^{+}$at $R(\mathrm{O}-\mathrm{Li})=4.0 \AA$ are reported in the lower portion of Table II. From the data calculated when $\mathrm{Li}^{+}$is replaced by a positive point charge, precluding the chance for $\mathrm{Li}$ orbitals to contaminate the $\mathrm{NH}_{3}$ system, it is clear from the last column of the table that correlation adds a repulsive component to the interaction. This repulsion is due primarily to the lowering of the $\mathrm{H}_{2} \mathrm{O}$ dipole moment by intramolecular correlation and the ensuing reduction in the
$\mathrm{H}_{2} \mathrm{O}-\mathrm{Li}^{+}$charge-dipole electrostatic interaction. Prior to removal of BSSE, the MP2 components are only slightly repulsive, and in the case of 6-31G** attractive. Subtraction of both primary and secondary BSSE leads to quite excellent agreement with the $\mathrm{H}_{2} \mathrm{O}$-point charge data, again suggesting removal of both of these terms (in full) would be appropriate in the general case. The SCF data in the preceding columns provides further support for this contention, since at either level, the $\mathrm{H}_{2} \mathrm{O}--\mathrm{Li}^{+}$interactions, even after correction for both $1^{\circ}$ and $2^{\circ} \mathrm{BSSE}$, are in no case more repulsive than the corresponding $\mathrm{H}_{2} \mathrm{O}-\mathrm{PC}$ (point charge) values.

\section{SUMMARY AND DISCUSSION}

In addition to the primary BSSE which has been well recognized for some time in the literature and which may be corrected by the counterpoise procedure, an additional artifact which we have focused on here is the secondary BSSE, due to the effect of ghost orbitals upon the calculated properties of each subunit. The magnitude of the $2^{\circ} \mathrm{BSSE}$ is in many cases comparable to or even larger than the primary BSSE and must likewise be removed for a correct description of any molecular interaction. Since the $1^{\circ}$ and $2^{\circ}$ BSSEs are often of the same sign, and since even when of opposite sign they behave differently with respect to intermolecular geometry, there is no reason to expect the $1^{\circ}$ and $2^{\circ}$ errors to cancel one another at either the SCF or MP2 level. Although the $2^{\circ}$ MP2 BSSE tends to be somewhat smaller in absolute magnitude than its SCF counterpart, the former is quite large in relation to the genuine MP2 contribution to the interaction; hence, failure to correct this error could severely distort our picture of the effects of electron correlation.

When first recognized several years ago as a potential difficulty, secondary BSSE was originally suggested to perhaps serve as an indirect mechanism whereby the accuracy of a calculation can be improved. ${ }^{32}$ The idea behind this hypothesis was that basis sets of limited size generally yield incorrect molecular properties, e.g., dipole moment and polarizability, which may be improved by the presence of the ghost orbitals of the partner subunit. We have an opportunity to test this hypothesis with the data we have generated here. Although the following points are made with specific reference to $\mathrm{H}_{3} \mathrm{~N}--\mathrm{Li}^{+}$, identical conclusions apply to $\mathrm{H}_{2} \mathrm{O}--\mathrm{Li}^{+}$, leading us to believe they are true in general for systems of this type.

We note first from Table I that most of the basis sets examined here overestimate the dipole moment of $\mathrm{NH}_{3}$, even after MP2 correction. However, inspection of Fig. 2 demonstrates that for a number of these sets, e.g., the standard $6-31 \mathrm{G}^{* *}$ and $6-311 \mathrm{G}^{* *}$, the exaggeration of the dipole moment is only further exacerbated by secondary BSSE. A second category contains the dif and $2 d$ basis sets which lead to rather accurate estimates of the dipole moment of isolated $\mathrm{NH}_{3}$ and hence could potentially provide a very good reproduction of the electrostatic portion of the interaction. This agreement is spoiled, however, by the increase in moment induced by the $\mathrm{Li}^{+}$ghost orbitals. The large negative secondary BSSEs associated with these basis sets (see Fig. 4) represent an error which progressively worsens as the two subunits approach one another. In both of these cases, the $2^{\circ}$ 
BSSE must be treated as an artifact which should be corrected where possible. The situation is somewhat different for the remaining basis sets (those containing + functions) where secondary BSSE generally lowers $\mu$ to values closer to the experimental quantity. However, even here, the situation is not so simple since the "improvement" of the moment is not constant but is instead a function of the distance of the ghost orbitals.

With regard to the polarizability of $\mathrm{NH}_{3}$, all basis sets underestimate this property by a substantial extent. The increases arising from secondary BSSE (see Fig. 3 ) can hence be expected to improve the appropriate component of the interaction energy to some degree. However, we are again faced with the dependence of the $2^{\circ}$ BSSE (due to $\alpha$ ) upon $R$. In summary, while the secondary BSSE may indeed improve certain aspects of the results in several cases, there are a number of obvious problems in treating this as a general rule.

The MP2 contribution to the interaction between two molecules may be conceptually partitioned into a number of components. The first is the dispersion energy arising from the mutual polarization of the two molecules. There are intramolecular terms as well, due to the effects of correlation upon the properties of each subunit. In addition to the preceding genuine factors, basis set superposition leads to contributions that are spurious and often sizable, which must be removed from the calculation. We reiterate that our data have provided no indication that subtraction of both the full counterpoise correction and the secondary BSSE leads to any overcorrection of the interaction. On the contrary, our fully corrected long-range MP2 interactions are slightly less repulsive than the true potential in which superposition is rigorously excluded by the substitution of a point charge for $\mathrm{Li}^{+}$. Similar conclusions apply to the SCF level where the $\mathrm{H}_{n} \mathrm{X}-\mathrm{Li}^{+}$interaction remains more attractive than the BSSE-free $\mathrm{H}_{n}$ X-PC energy, even after the former is corrected for both $1^{\circ}$ and $2^{\circ}$ errors.

In the systems examined here, the principal component of the secondary BSSE at long range arises from the effects of ghost orbitals upon the dipole moment of the neutral molecule. However, as the two subunits approach one another, the problem rapidly becomes further complicated as higherorder moments and polarizability play a more important role, as do penetration terms. Additional complexity results if the $\mathrm{Li}^{+}$is replaced by a nonspherical neutral molecule, as would occur in a $\mathrm{H}$ bond, and the properties of both molecules are subject to the influence of ghost orbitals. Because of the inherent difficulty in successfully removing all the components of secondary BSSE, it is strongly recommended that one should consider as a criterion for basis set selection an insensitivity of molecular properties to the presence of ghost orbitals. In this regard, we underscore the relatively small magnitudes of secondary BSSE associated with basis sets containing + functions, particularly if coupled to reoptimization of orbital exponents within the framework of the relevant molecules. The small primary BSSE associated with these basis sets ${ }^{27}$ is a second factor making them an attractive choice for studies of molecular interactions when computer limitations do not permit application of much larger sets.

\section{ACKNOWLEDGMENTS}

Some of the calculations were performed on the SIU Theoretical Chemistry Computer, funded in part by a grant from the Harris Corporation. This work was supported by grants from the National Institutes of Health (GM29391 and AM01059) and from the National Science Foundation (DMB-8612768). Partial support was provided by CPBP 01.12 from the Polish Academy of Science.

\footnotetext{
${ }^{1}$ A. Szabo and N.S. Ostlund, Modern Quantum Chemistry: Introduction to Advanced Electronic Structure Theory (MacMillan, New York, 1982); W. J. Hehre, L. Radom, P. v. R. Schleyer, and J. A. Pople, Ab Initio Molecular Orbital Theory (Wiley-Interscience, New York, 1986).

${ }^{2}$ S. F. Boys and F. Bernardi, Mol. Phys. 19, 553 (1970).

${ }^{3}$ P. Hobza, A. Mehlhorn, P. Ćársky, and R. Zahradnik, J. Mol. Struct. (Theochem) 138, 387 (1986).

${ }^{4}$ N. S. Ostlund and D. L. Merrifield, Chem. Phys. Lett. 39, 612 (1976).

${ }^{5}$ M. Bulski and G. Chalasinski, Theor. Chim. Acta 44, 399 (1977); J. H. van Lenthe, J. G. C. M. van Duijneveldt-van de Rijdt, and F. B. van Duijneveldt, Adv. Chem. Phys. (in press); L. M. J. Kroon-Batenburg and F. B. van Duijneveldt, J. Phys. Chem. 90, 5431 (1986); M. Gutowski, F. B. van Duijneveldt, G. Chalasinski, and L. Piela, Chem. Phys. Lett. 129, 325 (1986).
}

'S. J. Cole, K. Szalewicz, G. D. Purvis III, and R. J. Bartlett, J. Chem. Phys. 84, 6833 (1986).

${ }^{7}$ R. Cammi, R. Bonaccorsi, and J. Tomasi, Theor. Chim. Acta 68, 271 (1985).

${ }^{8}$ W. Kolos, Theor. Chim. Acta 54, 187 (1980); 51, 219 (1979)

${ }^{9}$ W. A. Sokalski, S. Roszak, P. C. Hariharan, and J. J. Kaufman, Int. J. Quantum Chem. 23, 847 (1983).

${ }^{10}$ L. Kurdi, E. Kochanski, and G. H. F. Diercksen, Chem. Phys. 92, 287 (1985).

"W. Meyer, P. C. Hariharan, and W. Kutzelnigg, J. Chem. Phys. 73, 1880 (1980).

${ }^{12}$ A. P. L. Rendell, G. B. Bacskay, and N. S. Hush, Chem. Phys. Lett. 117, 400 (1985).

${ }^{13}$ B. H. Wells and S. Wilson, Mol. Phys. 57, 421 (1986).

${ }^{14}$ S. M. Bachrach and A. Streitwieser, Jr., J. Am. Chem. Soc. 106, 2283 (1984).

${ }^{15}$ P. G. Burton and U. E. Senff, J. Chem. Phys. 76, 6073 (1982).

${ }^{16}$ D. M. Schwenke and D. G. Truhlar, J. Chem. Phys. 82, 2418 (1985).

${ }^{17}$ S. K. Loushin, S. Liu, and C. Dykstra, J. Chem. Phys. 84, 2720 (1986).

${ }^{18}$ P. Hobza, B. Schneider, P. Cársky, and R. Zahradnik, J. Mol. Struct. (Theochem) 138, 377 (1986); R. Zahradnik and P. Hobza, Int. J. Quantum Chem. 29, 663 (1986).

${ }^{19} \mathrm{G}$. Chalasinski and M. Gutowski, Mol. Phys. 57, 427 (1986); M. Gutowski, G. Chalasinski, and J. van Duijneveldt-van de Rijdt, Int. J. Quantum Chem. 26, 971 (1984); M. Gutowski, J. H. van Lenthe, J. Verbeek, F. B. van Duijneveldt, and G. Chalasinski, Chem. Phys. Lett. 124, 370 (1986); M. Bulski and G. Chalasinski, ibid. 128, 25 (1986).

${ }^{20} \mathrm{C}$. W. Bauschlicher, Jr., Chem. Phys. Lett. 122, 572 (1985)

${ }^{21}$ T. L. Mathers and N. R. Kestner, Int. J. Quantum Chem. Symp. 19, 297 (1986); M. D. Newton and N. R. Kestner, Chem. Phys. Lett. 94, 198 (1983).

${ }^{22}$ K. P. Sagarik, R. Ahlrichs, and S. Brode, Mol. Phys. 57, 1247 (1986).

${ }^{23}$ S. Roszak, W. A. Sokalski, P. C. Hariharan, and J. J. Kaufman, Theor. Chim. Acta 70, 81 (1986).

${ }^{24}$ G. H. F. Diercksen, V. Kellö, and A. J. Sadlej, Chem. Phys. 103, 55 (1986).

${ }^{25}$ B. H. Wells and S. Wilson, Mol. Phys. 50, 1295 (1983).

${ }^{26}$ M. M. Szczesniak and S. Scheiner, J. Chem. Phys. 84, 6328 (1986); Chem. Phys. Lett. 131, 230 (1986).

${ }^{27} \mathrm{Z}$. Latajka and S. Scheiner, J. Comput. Chem. (in press).

${ }^{28}$ Z. Latajka and S. Scheiner, Chem. Phys. Lett. 105, 435 (1984); Chem. Phys. 98, 59 (1985).

${ }^{29}$ J. O. Baum and J. L. Finney, Mol. Phys. 55, 1097 (1985).

${ }^{30}$ P. D. Dacre, Chem. Phys. Lett. 50, 147 (1977).

${ }^{31}$ S. L. Price and A. J. Stone, Chem. Phys. Lett. 65, 127 (1979).

${ }^{32}$ G. Karlström and A. J. Sadlej, Theor. Chim. Acta 61, 1 (1982).

${ }^{33}$ P. W. Fowler and A. D. Buckingham, Mol. Phys. 50, 1349 (1983). 
${ }^{34}$ P. D. Dacre, Mol. Phys. 36, 541 (1978).

${ }^{35}$ S. Tolosa Arroyo, J. E. Garcia, F. J. Olivares del Valle, and A. Requena, J. Mol. Struct. 136, 99 (1986).

${ }^{36}$ J. S. Binkley, R. A. Whiteside, R. Krishnan, R. Seeger, D. J. DeFrees, H. B. Schlegel, S. Topiol, L. R. Kahn, and J. A. Pople, QCPE Prog. No. 406 (1981).

${ }^{37}$ C. Moller and M.S. Plesset, Phys. Rev. 46, 618 (1934); J. S. Binkley and J. A. Pople, Int. J. Quantum Chem. 9, 229 (1975).

${ }^{38}$ A. J. Sadlej, J. Chem. Phys. 75, 320 (1981); Acta Phys. Pol. A 59, 669 (1981).

${ }^{39}$ G. H. F. Diercksen and A. J. Sadlej, J. Chem. Phys. 75, 1253 (1981).

${ }^{40}$ W. S. Benedict and E. K. Plyler, Can. J. Chem. 35, 1235 (1957).

${ }^{4}$ P. C. Hariharan and J. A. Pople, Theor. Chim. Acta 28, 213 (1973); Mol. Phys. 27, 209 (1974).

${ }^{42}$ J. G. C. M. van Duijneveldt-van de Rijdt and F.B. van Duijneveldt, J. Mol. Struct. 89, 185 (1982).

${ }^{43}$ R. Krishnan, J. S. Binkley, R. Seeger, and J. A. Pople, J. Chem. Phys. 72, 650 (1980).

${ }^{44}$ G. H. F. Diercksen and A. J. Sadlej, Mol. Phys. 57, 509 (1986).
${ }^{45}$ M. D. Marshall and J. S. Meunter, J. Mol. Spectrosc. 85, 322 (1981).

${ }^{46}$ G. D. Zeiss and W. J. Meath, Mol. Phys. 33, 1155 (1977).

${ }^{47}$ G. H. F. Diercksen, B. O. Roos, and A. J. Sadlej, Int. J. Quantum Chem. Symp, 17, 265 (1983); G. H. F. Diercksen, V. Kellö, and A. J. Sadlej, J. Chem. Phys. 79, 265 (1983); P. K. Mukherjee, T. Minato, and D. P. Chong, Int. J. Quantum Chem. 23, 447 (1983).

${ }^{48} \mathrm{The} 1^{\circ} \mathrm{BSSE}$ of $\mathrm{Li}^{+}$due to the $\mathrm{NH}_{3}$ ghost orbitals was computed and found to be negligible in all cases. Specifically, this term is less than 0.003 $\mathrm{kcal} / \mathrm{mol}$ for $R=1.5 \AA$ and dies off quickly with increasing distance.

${ }^{49} \mathrm{~J}$. J. Salzmann and C. K. Jorgensen, Helv. Chim. Acta 51, 1276 (1968).

${ }^{50}$ G. H. F. Diercksen and A. J. Sadlej, J. Chem. Phys. 75, 1253 (1981); H. Kistenmacher, H. Popkie, and E. Clementi, ibid. 59, 5892 (1973).

${ }^{31}$ K. P. Huber and G. Herzberg, Molecular Spectra and Molecular Structure (Van Nostrand Reinhold, New York, 1979), Vol. 4.

${ }^{52}$ G. H. F. Diercksen, V. Kellö, and A. J. Sadlej, J. Chem. Phys. 79, 2918 (1983).

${ }^{53}$ A. L. McClellan, Tables of Experimental Dipole Moments (Freeman, San Francisco, 1963).

${ }^{54}$ I. G. John, G. B. Bacskay, and N. S. Hush, Chem. Phys. 51, 49 (1980). 